\title{
Distributed observers with time-varying delays
}

\author{
Haik Silm, Rosane Ushirobira, Denis Efimov, Emilia Fridman, Jean-Pierre Richard, and Wim Michiels
}

\begin{abstract}
The distributed estimation problem is solved for continuous-time observer nodes that obtain real-time measurements but communicate with their neighbors over a communication network. To this end, the digital communication between the observer nodes is modeled by the time-delay approach where variable sampling intervals, transmission delays and packet dropouts are taken into account. An LMI for the design of the observer gains is derived using Halanay's inequality, the feasibility of which guarantees exponential stability with a selected convergence rate up to a maximum total delay. A comparison of the maximal delay on a numerical example shows the advantage of a distributed observer over a centralized one.
\end{abstract}

Index Terms-Distributed estimation problem, Time-varying delay, Networked control systems

\section{INTRODUCTION}

The continuous growth of information technology has enabled and created a strong necessity for decentralized control systems that can cope with the increasing demand for flexibility, scalability and performance. At the same time such a shift in the design paradigm raises new challenges related to networked systems, where effects of communication have to be taken into account. As such challenges we may cite datarate constraints and variations, delays, package dropouts and additional noises.

Together with decentralized control systems comes a growing interest in distributed estimation techniques [1]-[5]. The goal is to have state observers at sensor level who communicate only with neighboring observer nodes to fulfill the task of state estimation. This problem has been addressed from different view-points and most prevailing is the distributed Kalman filtering approach in discrete-time (see [6] for a recent survey). Often in these works, the effect of delays is not considered, while its presence may drastically influence the estimation performance (see [7], [8] for early works in discrete-time and [9], [10] for the constant-delay case).

This work has been partially supported by the project UCoCoS, funded by the European Union's Horizon 2020 research and innovation program under the Marie Skłodowska-Curie Grant Agreement No 675080.

H. Silm is with with the Department of Computer Science, KU Leuven, Celestijnenlaan 200A, 3001 Leuven, Belgium. He was previously with Univ. Lille, CNRS, Inria, Centrale Lille, UMR 9189 - CRIStAL, 59000 Lille, France (e-mail: haik.silm@kuleuven.be).

R. Ushirobira is with Inria, Univ. Lille, CNRS, UMR 9189 - CRIStAL, 59000 Lille, France (e-mail: rosane.ushirobira@inria.fr).

D. Efimov is with Inria, Univ. Lille, CNRS, UMR 9189 - CRIStAL, 59000 Lille, France and with ITMO University, 197101 Saint Petersburg, Russia (denis.efimov@inria.fr).

E. Fridman is with the Department of Electrical Engineering and Systems, Tel Aviv University, 69978 Tel Aviv, Israel (e-mail:emilia@tauex.tau.ac.il).

J.-P. Richard is with Univ. Lille, CNRS, Inria, Centrale Lille, UMR 9189 - CRIStAL, 59000 Lille, France (jean-pierre.richard@centralelille.fr).

W. Michiels is with the Department of Computer Science, KU Leuven, Celestijnenlaan 200A, 3001 Leuven, Belgium (e-mail: wim.michiels@cs.kuleuven.be).
In the present work, we keep a continuous-time framework, while considering the various delays which can occur in a communication network. In such a case an effective method is the time-delay approach [11], where for example the sampled-data nature of digital communication is model by a sawtooth shaped time-varying delay. In the context of distributed estimation this has been previously used in [12] specifically for a Round-Robin protocol. The advantage is that different sampling-times can be better modeled and it allows to use a high-rate of sampling for the plant output with low communication rates between the observer nodes as proposed in [13].

This also provides a good motivation for distributed observers which use a consensus-term, since, as we will show, in comparison with a centralized observer it can have better robustness with respect to delays, by shifting the delay from the measurement input to the communication. This has been preliminary presented in [14], but for constant delays.

The time-delay approach for the stability analysis leads to a constructive design formulated in terms of an LMI. For distributed observers, an application of this method is novel, and to simplify the presentation we start with the delay-free case. We prove that the LMIs for the design are feasible under standard nonrestrictive conditions in the delay-free case and they will form the basis for the time-varying delay case. External disturbances and measurement noise can also be considered in the LMI framework; however, for the sake of comparison we will consider the exponential convergence rate of the nominal system as design criteria.

The outline of the paper is as follows. First the problem setting of distributed observers is introduced and we contrast it with the standard centralized setting. Then the known LMIbased design for systems with time-varying delay is presented for a centralized observer. Next, we introduce the distributed observer setting and provide a new method for the design in the delay-free case. The main result consists in a design for the distributed observer, where time-varying delays are taken into account using the above methods. Finally, a comparison is made between a centralized and distributed observer in terms of the bound of the time-varying delay under a specified minimal convergence rate.

\section{Problem Setting}

Consider a (large-scale) linear time-invariant plant with multiple outputs

$$
\dot{x}(t)=A x(t), \quad y_{i}(t)=C_{i} x(t),
$$

$i=1, \ldots, N$, with state vector $x \in \mathbb{R}^{n}$ and dynamics matrix $A \in \mathbb{R}^{n \times n}$, which is observed by a set of $N$ sensor nodes, each corresponding to one of the outputs $y_{i} \in \mathbb{R}^{m_{i}}$ with output 
matrix $C_{i} \in \mathbb{R}^{m_{i} \times n}$. The goal is to reconstruct the full state of the plant at sensor level from the measurements, under the constraint that this is only possible using all the outputs, i.e. only the pair $\left(A, \operatorname{col}\left\{C_{i}\right\}_{i=1}^{N}\right)$ is observable but any subset of the outputs is not. In this paper $\operatorname{col}\left\{C_{i}\right\}_{i=1}^{N} \in \mathbb{R}^{m \times n}$, where $m=\sum_{i=1}^{N} m_{i}$, denotes a block column matrix formed of the sequence of matrices $C_{1}, \ldots, C_{N}$, and correspondingly row $\left\{B_{i}\right\}_{i=1}^{N}$ denotes a block row matrix and $\operatorname{diag}\left\{A_{i}\right\}_{i=1}^{N}$ a block diagonal matrix (occasionally abbreviated with $\bar{A}$ ).

\section{A. Observer concepts}

In the centralized setting all the measurements from the sensors are gathered at a central entity and the reconstructed state estimate $\tilde{x}$ is transmitted back, see Fig. 1a. In the distributed case, each sensor will act directly as an observer node with a local state estimate $\tilde{x}_{i}, i=1, \ldots, N$. Since it is not possible to reconstruct the state from a single output $y_{i}$ alone, the observer nodes have to exchange information with the other nodes. A straightforward approach would be to implement each sensor node as a centralized observer and to distribute the individual measurements between each of them, however this would require all-to-all communication because all outputs are necessary to reconstruct the state. Instead, as we will present in Section III-B, the observers nodes will only exchange their local state estimates over a communication graph which depends on the spatial distribution of the sensors or other considerations, see Fig. $1 \mathrm{~b}$

A first comparison of both concepts shows as an advantage for the distributed setting that the communication is only local, while the transmission of the measurements to the centralized observer and the return of the state estimate is possibly impacted by large delays.

\section{B. Features of communication}

We pose ourselves now the question, whether the relative performance of the two structures may additionally differ due to what is communicated. Assuming that the estimation algorithms are implemented on fast processors (compared to the plant's dynamics), the dynamics of the observers can be described as continuous-time systems. At the same time, communication resources in sensor networks are typically limited, so significant delays and data rate constraints are inevitable. Note that we do not account for delays from the plant output to the nodes (either the sensor nodes are placed close to the corresponding plant output or there is no difference in the effect for both observer structures.)

We assume that all the sensor nodes and observers have synchronized clocks and know the delay (e.g., using a network time protocol and including time-stamps in the transmission). Between a transmission interval the continuous centralized observer or the observer nodes will make the incoming information continuous by holding their last sample. This corresponds to a time-varying delay with a sawtooth like shape in the signal. The resulting aberration puts a limit on the maximum transmission interval and delay, which in this work we compare for both structures on a numerical example by looking at the maximum $\bar{\tau}$ of the time-varying delay. Note

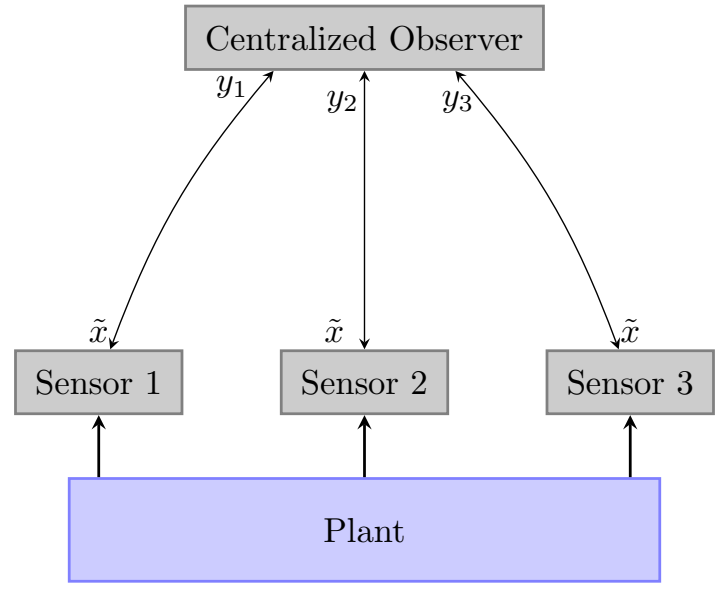

(a) Centralized

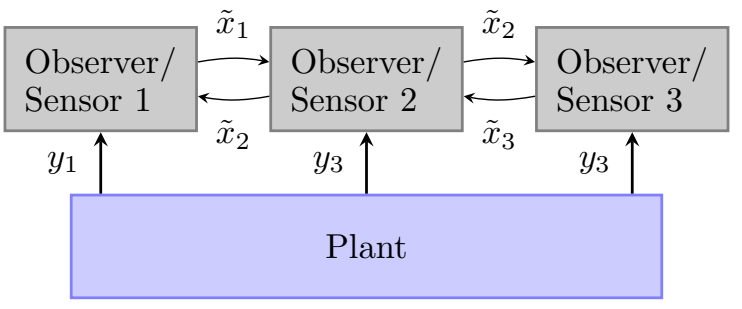

(b) Distributed

Fig. 1: Examples of the two observer structures for $N=3$.

that using a time-varying delay, we can also model other network induced effects (computation delay, communication delay, synchronization of data streams, etc.)

\section{Performance measure}

As it is the case for the delay-margin [14], it might be not enough to optimize and compare the design only with respect to a maximal delay, since the resulting performance can be quiet different in terms of convergence rate. Therefore, we will additionally require the same minimal exponential convergence rate. In the following we denote by $x_{t}$ the function $x(t)$ on the interval $[t-\bar{\tau}, t]$, by $C_{[-\bar{\tau}, 0]}$ the class of such a continuous functions and by $W_{[-\bar{\tau}, 0]}$ a subclass of functions having a square integrable derivative with respect to time.

Definition 1. A system $\dot{x}(t)=f\left(x_{t}\right)$ is said to be exponentially stable with a convergence rate $\alpha>0$, if for any initial condition $x_{0} \in W_{[-\bar{\tau}, 0]}$ the solution satisfies

$$
|x(t)| \leq c \mathrm{e}^{-\alpha t}\left\|x_{0}\right\|_{W}
$$

with a constant $c \geq 1$, where $\left\|x_{t}\right\|_{W}=\max _{t \in[t-\bar{\tau}, t]}|x(t)|+$ $\sqrt{\int_{t-\bar{\tau}}^{t}|\dot{x}(s)|^{2} \mathrm{~d} s}$.

To establish exponential convergence rate we will use in the centralized case and in the distributed undelayed case a form of the comparison lemma [15]. For the distributed observer with time-varying delays we will use Halanay's inequality ([16, p. 138], [17, p. 378]): 
Lemma 1. Let $V:[-\bar{\tau}, \infty] \rightarrow \mathbb{R}_{+}$be a bounded function on $[-\bar{\tau}, 0]$, absolutely continuous on $[0, \infty)$. If for some $0<$ $\delta_{1}<\delta_{0}$, the inequality

$$
\dot{V}(t)+2 \delta_{0} V(t)-2 \delta_{1} \sup _{-\bar{\tau} \leq \theta \leq 0} V(t+\theta) \leq 0
$$

holds for all $t \geq 0$, then

$$
V(t) \leq \mathrm{e}^{-2 \delta t} \sup _{-\bar{\tau} \leq \theta \leq 0} V(\theta)
$$

where $\delta$ is the solution of

$$
\delta-\delta_{0}+\delta_{1} \exp ^{2 \delta \bar{\tau}}=0 .
$$

\section{OBSERVER DESIGN}

In this section, we recall the observer design in the presence of time-varying delays in the centralized setting. Then the design of a distributed observer in the delay-free case is given. Together these results form the basis of the main development of this work: a distributed observer design for time-varying delays. A comparison of the centralized and distributed observers is carried out with simulations using a sawtooth shaped delay in the next section.

\section{A. Centralized observer}

For the sake of convenience we will assume that the communication rates and transmission delays from the sensor nodes to the centralized observer are identical so that the individual measurement outputs of the $N$ sensors can be combined to $y=\left[\begin{array}{lll}y_{1}^{\top} & \ldots & y_{N}^{\top}\end{array}\right]^{\top}=C x$, where $C=\operatorname{col}\left\{C_{i}\right\}_{i=1}^{N}$. For $k \in \mathbb{N}$, let $t_{k}$ denote the time instances when the observer receives an update from the sensors and $\eta_{k}$ the transmission delays. The observer is then implemented as a Luenberger observer, where the output-injection term is kept constant during a transmission interval and the delay is accounted for

$$
\dot{\tilde{x}}(t)=A \tilde{x}(t)-L\left(C \tilde{x}\left(t_{k}-\eta_{k}\right)-y\left(t_{k}-\eta_{k}\right)\right),
$$

$t_{k} \leq t<t_{k+1}$, where $\tilde{x} \in \mathbb{R}^{n}$ is the estimate of the state $x$ and $L$ is the observer gain to be designed. The error $e(t)=$ $\tilde{x}(t)-x(t)$ leads to the dynamics

$$
\dot{e}(t)=A e(t)-L C e\left(t_{k}-\eta_{k}\right), \quad t_{k} \leq t<t_{k+1},
$$

which equivalently can be rewritten as

$$
\dot{e}(t)=A e(t)-L C e(t-\tau(t))
$$

with a piecewise linear time-varying delay $\tau(t)$, where $\dot{\tau}(t)=$ 1 and its maximum is denoted as $\bar{\tau}=\max _{k}\left\{t_{k+1}-t_{k}+\eta_{k}\right\}$, the maximum of the sum of the transmission interval and delay.

To obtain a sufficient upper bound of $\bar{\tau}$ for a given convergence speed, an LMI is derived below to select a gain $L$ that guarantees exponential stability of the error system for the highest possible $\bar{\tau}$.

Following [16], we take as a Lyapunov-Krasovskii functional for systems with fast-varying delays and exponential convergence

$$
\begin{aligned}
& V\left(t, e_{t}, \dot{e}_{t}\right)=e^{\top}(t) P e(t)+\int_{t-\bar{\tau}}^{t} \mathrm{e}^{-2 \alpha(t-s)} e^{\top}(s) S e(s) \mathrm{d} s \\
&+\bar{\tau} \int_{t-\bar{\tau}}^{t} \mathrm{e}^{-2 \alpha(t-s)}(\bar{\tau}+s-t) \dot{e}^{\top}(s) \operatorname{Re}(s) \mathrm{d} s
\end{aligned}
$$

where $P>0, R>0$ and $S>0$. It can be shown that there exist constants $\bar{k}>0$ and $\underline{k}>0$ so that $\underline{k}|e(t)|^{2} \leq$ $V\left(t, e_{t}, \dot{e}_{t}\right) \leq \bar{k}\left\|e_{t}\right\|_{W}^{2}$ for all $t \geq 0$ and $e_{t} \in W_{[-\bar{\tau}, 0]}$. Taking the derivative and using the Leibniz integral rule lead to

$$
\begin{gathered}
\dot{V}+2 \alpha V=2 \dot{e}^{\top}(t) P e(t)+e^{\top}(t)(2 \alpha P+S) e(t) \\
-\mathrm{e}^{-2 \alpha \bar{\tau}} e^{\top}(t-\bar{\tau}) S e(t-\bar{\tau}) \\
+\bar{\tau}^{2} \dot{e}^{\top}(t) \operatorname{Re}(t)-\bar{\tau} \int_{t-\bar{\tau}}^{t} \mathrm{e}^{-2 \alpha(t-s)} \dot{e}^{\top}(s) \operatorname{Re}(s) \mathrm{d} s
\end{gathered}
$$

and after using $\mathrm{e}^{-2 \alpha(t-s)} \geq \mathrm{e}^{-2 \alpha \bar{\tau}}$ for $s \in[t-\bar{\tau}, t]$ the following lemma is applied for the remaining integral:

Lemma 2 ([16], [18]). Under assumption of

$$
\left[\begin{array}{cc}
R & S_{12} \\
* & R
\end{array}\right] \geq 0
$$

for some $S_{12}$ the following inequality holds:

$$
\begin{aligned}
& -\bar{\tau} \int_{t-\bar{\tau}}^{t} \dot{e}^{\top}(s) R \dot{e}(s) \mathrm{d} s \\
\leq & {\left[\begin{array}{c}
e(t) \\
e(t-\bar{\tau}) \\
e(t-\tau(t))
\end{array}\right]^{\top}\left[\begin{array}{ccc}
-R & S_{12} & R-S_{12} \\
* & -R & R-S_{12} \\
* & * & -R+S_{12}+S_{12}^{\top}
\end{array}\right]\left[\begin{array}{c}
e(t) \\
e(t-\bar{\tau}) \\
e(t-\tau(t))
\end{array}\right] . }
\end{aligned}
$$

In line with the descriptor method [16] the system dynamics is incorporated by adding

$$
\left(P_{2} e(t)+P_{3} \dot{e}(t)\right)^{\top}(A e(t)-L C e(t-\tau(t))-\dot{e}(t))=0
$$

to (5), where $P_{2}$ and $P_{3}$ are auxiliary matrices. Together with comparison lemma this leads to an estimate

$$
\dot{V}+2 \alpha V \leq \eta^{\top}(t) \Phi \eta(t)
$$

where $\eta(t)=\operatorname{col}\{e(t), \dot{e}(t), e(t-\bar{\tau}), e(t-\tau(t))\}, \Phi=$

$$
\left[\begin{array}{cccc}
\Phi_{11} & P-P_{2}^{\top}+A^{\top} P_{3} & \mathrm{e}^{-2 \alpha \bar{\tau}} S_{12} & -P_{2}^{\top} L C+\mathrm{e}^{-2 \alpha \bar{\tau}}\left(R-S_{12}\right) \\
* & -P_{3}-P_{3}^{\top}+\bar{\tau}^{2} R & 0 & -P^{\top} L C \\
* & * & -\mathrm{e}^{-2 \alpha \bar{\tau}}(S+R) & \mathrm{e}^{-2 \alpha \bar{\tau}}\left(R-S_{12}\right) \\
* & * & * & \mathrm{e}^{-2 \alpha \bar{\tau}}\left(-2 R+S_{12}+S_{12}^{\top}\right)
\end{array}\right],
$$

$\Phi_{11}=A^{\top} P_{2}+P_{2}^{\top} A+2 \alpha P+S-\mathrm{e}^{-2 \alpha \bar{\tau}} R$, for which the feasibility of the LMI $\Phi \leq 0$ implies exponential stability, since

$$
\underline{k}|e(t)|^{2} \leq V\left(t, e_{t}, \dot{e}_{t}\right) \leq \mathrm{e}^{-2 \alpha t} V\left(0, e_{0}, \dot{e}_{0}\right) \leq \bar{k}\left\|e_{0}\right\|_{W}^{2},
$$

where $\underline{k}=\lambda_{\min }(P)$ is the minimal eigenvalue of the matrix $P$. Then to design the gain $L$, we make the substitution $P_{3}=\epsilon P_{2}$ with some $\epsilon>0$ and $Y=P_{2}^{\top} L$ to obtain the following result:

Proposition 1. Given $\bar{\tau}>0, \alpha>0$ and a tuning variable $\epsilon>0$, let there exist matrices $P>0, S>0, R>0, P_{2}, Y, S_{12}$ that satisfy (6) and the $L M I \Phi \leq 0$ with $\Phi=$

$$
\left[\begin{array}{cccc}
\Phi_{11} & P-P_{2}^{\top}+\epsilon A^{\top} P_{2} & \mathrm{e}^{-2 \alpha \bar{\tau}} S_{12} & -Y C+\mathrm{e}^{-2 \alpha \bar{\tau}}\left(R-S_{12}\right) \\
* & -\epsilon\left(P_{2}+P_{2}^{\top}\right)+\bar{\tau}^{2} R & 0 & -\epsilon Y C \\
* & * & -\mathrm{e}^{-2 \alpha \bar{\tau}}(S+R) & \mathrm{e}^{-2 \alpha \bar{\tau}}\left(R-S_{12}\right) \\
* & * & * & \mathrm{e}^{-2 \alpha \bar{\tau}}\left(-2 R+S_{12}+S_{12}^{\top}\right)
\end{array}\right],
$$

then the error of the observer (3) for the system (1) with gain $L=\left(P_{2}^{\top}\right)^{-1} Y$ is guaranteed to converge exponentially with the rate $\alpha$ for any delay $0<\tau(t)<\bar{\tau}$.

Since the LMIs above provide a sufficient condition, the convergence rate might be preserved also for larger delays. 
Moreover, to obtain less conservative estimates, the LMI for analysis (8) can be used a posteriori (i.e. designing the observer by the theorem, then fixing the gains and determining guaranteed lower bound by the analysis LMIs).

Remark 1. In the case of different communication rates of the sensors, the error system is $\dot{e}(t)=A e(t)-\sum_{i=1}^{N} L_{i} C_{i} e(t-$ $\left.\tau_{i}(t)\right)$ with different $\tau_{i}(t)$ and $\bar{\tau}_{i}$ and we would use additional integral terms in (4) for each sensor. Such a modification is omitted for brevity of presentation.

\section{B. Distributed observer: Delay-free case}

In the distributed setting, an observer node is implemented on each sensor. Since the pairs $\left(A, C_{i}\right)$ are not observable, the measurement only does not suffice to reconstruct the state. Therefore, following [19], the output-injection is complemented by a consensus-term to use the information provided by the other nodes in a distributed fashion. We denote by $\mathcal{N}_{i}$ the set of nodes who provide their estimate to node $i$ (e.g. for the example in Fig. $1 \mathrm{~b}, \mathcal{N}_{2}=\{1,3\}$ ). In this subsection we will assume that there is no sampling and no delay in the communication of the observers. The estimate at the $i$ th observer node $\tilde{x}_{i}$ is then obtained as

$$
\begin{aligned}
\dot{\tilde{x}}_{i}(t)=A \tilde{x}_{i}(t)-L_{i}\left(C_{i} \tilde{x}_{i}(t)-y_{i}(t)\right) & \\
& -\sum_{j \in \mathcal{N}_{i}} H_{i, j}\left(\tilde{x}_{i}(t)-\tilde{x}_{j}(t)\right), i=1, \ldots, N,
\end{aligned}
$$

where $L_{i}$ are the local observer gains and $H_{i, j}$ are the consensus gains with the neighboring nodes, which have to be designed.

With the observer error for each node $e_{i}=\tilde{x}_{i}-x$ we are now interested in the stability of the coupled error systems

$$
\dot{e}_{i}(t)=A e_{i}(t)-L_{i} C_{i} e_{i}(t)-\sum_{j \in \mathcal{N}_{i}} H_{i, j}\left(e_{i}(t)-e_{j}(t)\right) .
$$

Looking at the combined error $E=\left[\begin{array}{lll}e_{1}^{\top} & \ldots & e_{N}^{\top}\end{array}\right]^{\top}$ this corresponds to the stability of the system

$$
\dot{E}(t)=\bar{A} E(t)-\overline{L C} E(t)-\mathcal{H} E(t),
$$

where $\bar{A}=I_{N} \otimes A, \bar{C}=\operatorname{diag}\left\{C_{i}\right\}_{i=1}^{n}, \bar{L}=\operatorname{diag}\left\{L_{i}\right\}_{i=1}^{n}$ and

$$
\mathcal{H}=\left[\begin{array}{cccc}
\sum_{j \in \mathcal{N}_{1}} H_{1, j} & -H_{1,2} & \ldots & -H_{1, N} \\
-H_{N, 1} & \ldots & -H_{N, N-1} & \sum_{j \in \mathcal{N}_{N}} H_{N, j}
\end{array}\right]
$$

(here $H_{i, j}=0$ if $j \notin \mathcal{N}_{i}$ ). The exponential stability with a convergence rate $\alpha>0$ of (11) can be analyzed by a quadratic Lyapunov function $W=E^{\top} P E$ giving

$$
\overline{P A}+\bar{A}^{\top} \bar{P}-\overline{P L C}-\bar{C}^{\top} \bar{L}^{\top} \bar{P}-\bar{P} \mathcal{H}-\mathcal{H}^{\top} \bar{P}+\alpha \bar{P}<0 .
$$

To design the consensus gains $H_{i, j}$ we have to assume a blockdiagonal structure of the matrix $\bar{P}=\operatorname{diag}\left\{P_{i}\right\}_{i=1}^{N}$ (this means that the system can be designed to be block-diagonally stable
[20]) leading to an LMI $\Phi<0, \Phi \in \mathbb{R}^{n N \times n N}$, with diagonal entries

$$
\begin{aligned}
\Phi_{i i}=P_{i} A+A^{\top} P_{i}-P_{i} L_{i} C_{i} & -C_{i}^{\top} L_{i}^{\top} P_{i}^{\top} \\
& -P_{i} \sum_{j \in \mathcal{N}_{i}} H_{i, j}-\sum_{j \in \mathcal{N}_{i}} H_{i, j}^{\top} P_{i}
\end{aligned}
$$

and off-diagonal entries $\Phi_{i j}=\Phi_{i, j}^{\top}=P_{i} H_{i, j}+P_{j} H_{j, i}$. The substitution $Z_{i, j}=P_{i} H_{i, j}$ and $Y_{i}=P_{i} C_{i}$ then leads to an LMI for the design. The following lemma establishes that the LMI is feasible under non-restrictive conditions (joint observability and strong connectedness).

Lemma 3. If the pair $\left(A, \operatorname{col}\{C\}_{i=1}^{N}\right)$ is observable and the communication graph is strongly connected then there exist $L_{i}$ and $H_{i, j}$ such that the dynamics of the error of the observer network (11) admits a block-diagonal Lyapunov-matrix $\bar{P}=$ $\operatorname{diag}\left\{P_{i}\right\}_{i=1}^{N}$.

Proof. Selecting $H_{i, j}=\alpha T_{u, i} T_{u, i}^{\top}$ and $L_{i}=T_{o, i} L_{o, i}$, where the columns of $T_{u, i}$ form a basis of the kernel of the observability matrix of the pair $A$ and $C_{i}$, and $T_{i}=$ $\left[\begin{array}{ll}T_{o, i} & T_{u, i}\end{array}\right]$ is an orthogonal matrix, then the dynamics of the error of the network (11) can be transformed with $E=$ $\left[\operatorname{diag}\left\{T_{o, i}\right\}_{i=1}^{N} \operatorname{diag}\left\{T_{u, i}\right\}_{i=1}^{N}\right]\left[\begin{array}{c}E_{o} \\ E_{u}\end{array}\right]$ into block-triangular form

$$
\left[\begin{array}{l}
\dot{E}_{o} \\
\dot{E}_{u}
\end{array}\right]=\left[\begin{array}{cc}
\overline{A_{o}}-\overline{L_{o} C_{o}} & 0 \\
\overline{A_{r}}+\alpha{\overline{T_{u}}}^{\top} \mathcal{A} \overline{T_{o}} & \overline{A_{u}}-\alpha{\overline{T_{u}}}^{\top}(\mathcal{L} \otimes I) \overline{T_{u}}
\end{array}\right]\left[\begin{array}{l}
E_{o} \\
E_{u}
\end{array}\right],
$$

where $A_{o}=T_{o, i}^{T} A T_{o, i}, A_{u}=T_{u, i}^{T} A T_{u, i}, A_{r}=T_{u, i}^{T} A T_{o, i}$, $C_{o}=C T_{o, i}$, and $\mathcal{A}$ and $\mathcal{L}$ are the adjacency and Laplacian matrix of the communication graph, respectively.

Since it is block-triangular it has a Lyapunov matrix of the form $\tilde{P}=\left[\begin{array}{cc}\operatorname{diag}\left\{P_{o, i}\right\}_{i=1}^{N} & 0 \\ 0 & \gamma \operatorname{diag}\left\{P_{u, i}\right\}_{i=1}^{N}\end{array}\right]$ with some $\gamma>0$ if $\operatorname{diag}\left\{P_{o, i}\right\}_{i=1}^{N}$ and $\operatorname{diag}\left\{P_{u, i}\right\}_{i=1}^{N}$ are Lyapunov matrices for $\overline{A_{o}}-\overline{L_{o} C_{o}}$ and $\overline{A_{u}}-\alpha{\overline{T_{u}}}^{\top}(\mathcal{L} \otimes I) \overline{T_{u}}$, respectively. The former is obvious and for the latter we can use the fact that $\bar{T}_{u}^{\top}(\mathcal{L} \otimes I) \overline{T_{u}}$ admits a Lyapunov matrix which is diagonal under the conditions of the lemma (see [21]) and select $\alpha>0$ sufficiently large. Returning to original coordinates, the Lyapunov-matrix is transformed as

$$
\begin{aligned}
& \bar{P}=\left[\operatorname{diag}\left\{T_{o, i}\right\}_{i=1}^{N} \quad \operatorname{diag}\left\{T_{u, i}\right\}_{i=1}^{N}\right] \tilde{P}\left[\begin{array}{l}
\operatorname{diag}\left\{T_{o, i}^{\top}\right\}_{i=1}^{N} \\
\operatorname{diag}\left\{T_{u, i}^{\top}\right\}_{i=1}^{N}
\end{array}\right] \\
& =\left[\begin{array}{cc}
\operatorname{diag}\left\{T_{o, i} P_{o, i} T_{o, i}^{\boldsymbol{\top}}\right\} & 0 \\
0 & \operatorname{diag}\left\{T_{u, i} P_{u, i} T_{u, i}^{\boldsymbol{\top}}\right\}
\end{array}\right] .
\end{aligned}
$$

Note that the choice of $\bar{P}$ implies a Lyapunov function $W=\sum_{i=1}^{N} V_{i}$ with $V_{i}=e_{i}^{\top} P_{i} e_{i}$. Inspired by this and [12] we can then deduce the stability of the coupled systems with individual Lyapunov functions $\left(\sum_{j: i \in \mathcal{N}_{j}}\right.$ denotes the sum over the nodes which use the estimate of node $i$ ): 
Lemma 4. For $i=1, \ldots, N$, assume there exist Lyapunov functions $\underline{k}\left|e_{i}\right|^{2} \leq V_{i}\left(e_{i}\right) \leq \bar{k}\left|e_{i}\right|^{2}$ with $\underline{k}>0$ and $\bar{k}>0$, $\alpha_{i}>0$ and $\beta_{i, j}>0, j \in \mathcal{N}_{i}$, for which the inequalities

$$
\dot{V}_{i}+2 \alpha_{i} V_{i}-\sum_{j \in \mathcal{N}_{i}} \beta_{i, j} V_{j} \leq 0
$$

hold with

$$
\delta=\min _{i}\left(2 \alpha_{i}-\sum_{j: i \in \mathcal{N}_{j}} \beta_{j, i}\right)>0 .
$$

Then the systems $(10)$ are exponentially stable with a convergence rate $\delta$.

Proof. Taking the Lyapunov function $W=\sum_{i=1}^{N} V_{i}$ we obtain (by exchanging indices)

$$
\dot{W}=\sum_{i=1}^{N} \dot{V}_{i} \leq-\sum_{i=1}^{N}\left(2 \alpha_{i}-\sum_{j: i \in \mathcal{N}_{j}} \beta_{j, i}\right) V_{i} \leq-2 \delta W .
$$

From the comparison lemma we can then conclude exponential stability.

This leads to another formulation of LMIs for the design of a distributed observer in the delay-free case:

Theorem 1. For $i=1, \ldots, N$, given $\alpha_{i}>0$ and $\beta_{i, j}>0$ such that $\sum_{j: i \in \mathcal{N}_{j}} \beta_{j, i}<2 \alpha_{i}$, assume there exist matrices $P_{i}>0$ and $Z_{i, j}, j \in \mathcal{N}_{i}$ for which the system of LMIs $\Phi_{i} \leq 0$ with

$$
\Phi_{i}=\left[\begin{array}{cc}
\Phi_{1, i} & \operatorname{row}\left\{Z_{i, j}\right\}_{j \in \mathcal{N}_{i}} \\
* & -\operatorname{diag}\left\{\beta_{i, j} P_{j}\right\}_{j \in \mathcal{N}_{i}}
\end{array}\right],
$$

and $\Phi_{1, i}=A^{\top} P_{i}+P_{i} A-C_{i}^{\top} Y_{1, i}^{\top}-Y_{1, i} C_{i}-\sum_{j \in \mathcal{N}_{i}} Z_{i, j}-$ $\sum_{j \in \mathcal{N}_{i}} Z_{i, j}^{\top}+2 \alpha_{i} P_{i}$ is feasible. Then the distributed observer (9) for the system (1) with gains $L_{i}=P_{i}^{-1} Y_{i}$ and $H_{i . j}=$ $P_{i}^{-1} Z_{i, j}$ converges exponentially with rate $\delta$ given by 15 .

Proof. Taking the Lyapunov functions $V_{i}=e_{i}^{\top} P_{i} e_{i}$, the inequality (14) with

$$
\begin{aligned}
& \dot{V}_{i}=e_{i}^{\top}\left(P_{i}\left(A_{i}-L_{i} C_{i}-\sum_{j \in \mathcal{N}_{i}} H_{i, j}\right)\right. \\
& \left.+\left(A_{i}-L_{i} C_{i}-\sum_{j \in \mathcal{N}_{i}} H_{i, j}\right)^{\top} P_{i}\right) e_{i}+2 e_{i}^{\top} P_{i} \operatorname{row} \underset{\substack{\left\{\mathcal{N}_{i} \\
j \in \mathcal{N}_{i}\right.}}{H_{i}},
\end{aligned}
$$

where $\mathbf{e}_{i}=\operatorname{col}\left\{e_{j}\right\}_{j \in \mathcal{N}_{i}}$ and $-\sum_{j \in \mathcal{N}_{i}} \beta_{i, j} V_{j} \leq$ $-\mathbf{e}_{i}^{\top} \operatorname{diag}\left\{\beta_{i, j} P_{j}\right\}_{j \in \mathcal{N}_{i}} \mathbf{e}_{i}$ yields

$$
\dot{V}+2 \alpha V-\sum_{j \in \mathcal{N}_{i}} \beta_{i, j} V_{j} \leq\left[\begin{array}{c}
e_{i} \\
\mathbf{e}_{i}
\end{array}\right]^{\top} \Phi_{i}\left[\begin{array}{l}
e_{i} \\
\mathbf{e}_{i}
\end{array}\right] \leq 0 .
$$

Then according to Lemma 4 the error system $(10)$ is exponentially stable if $\Phi_{i} \leq 0, i=1, \ldots, N$.

Note that the neighboring relation is included in the last term of (14), i.e. we take the sum over all neighbors.

Remark 2. The inequalities (14) have another interpretation when considering the vector $\mathbf{V}=\left[\begin{array}{lll}V_{1} & \ldots & V_{N}\end{array}\right]^{\top}$. They imply $\dot{\mathbf{V}} \leq M \mathbf{V}$ with diagonal-dominant Metzler matrix $M$ where $M_{i i}=-\alpha_{i, j}, \quad M_{i j}=\beta_{i, j}$. This means $M$ is HurwitzMetzler for which the following Lyapunov equation is fulfilled:

$$
p^{\top} M<-q^{T},
$$

with vectors $p \in \mathbb{R}^{N}$ and $q \in \mathbb{R}^{N}$ with strictly positive entries. Then the Lyapunov function $W=p^{T} \mathbf{V}$ will lead to

$$
\dot{W}<-q^{\top} V<-\delta W
$$

for some $\delta>0$ (since there exists a $\delta$ for which $\delta p^{\top}$ is element-wise smaller than $q^{\top}$ ). Therefore, by considering such a Lyapunov function the constraints $\sum_{j: i \in \mathcal{N}_{j}} \beta_{j, i}<2 \alpha_{i}$ can be relaxed.

Compared to the centralized LMI (12), the system of LMIs of Theorem 1 might be used as an approach to decentralize the design of the gains, by introducing the condition $P_{i}>I$ for all $i=1, \ldots, N$. Then at each observer node the individual LMI

$$
\left[\begin{array}{cc}
\Phi_{1, i} & \operatorname{row}\left\{Z_{i, j}\right\}_{j \in \mathcal{N}_{i}} \\
* & -\operatorname{diag}\left\{\beta_{i, j} I\right\}_{j \in \mathcal{N}_{i}}
\end{array}\right]<0
$$

can be solved for $\beta_{i, j}>0$, from which $\Phi_{i}<0$ from (16) follows. Then in a second step the nodes have to send their $\beta_{i, j}$ to their neighbors $\mathcal{N}_{i}$ to check the condition $\left[-\alpha_{i} \quad \operatorname{row}\left\{\beta_{j, i}\right\}_{j \in \mathcal{N}_{i}}\right] p<q_{i}$, with a positive vector $p$ and $q_{i}>0$.

\section{Distributed observer: Time-varying delay}

In the previous section the communication between the nodes as well as the measurement inputs were supposed to be almost instantaneous. For the measurement inputs this is a reasonable assumption since each observer node is implemented on the corresponding sensor and hence they are available in real-time. However, since the sensors are distributed over the plant, the communication lag between the sensors can be significant and moreover constrained in their rate.

Compared to 10$]$ the consensus term is then modified to take into account the delayed sampled-data communication leading to the error systems

$$
\begin{aligned}
\dot{e}_{i}(t)= & \left(A-L_{i} C_{i}\right) e_{i}(t) \\
& -\sum_{j \in \mathcal{N}_{i}} H_{i, j}\left(e_{i}\left(t-\tau_{i}(t)\right)-e_{j}\left(t-\tau_{i}(t)\right)\right),
\end{aligned}
$$

$i=1, \ldots, N$, with the time-varying delay $\tau_{i}(t)$ and $\bar{\tau}=$ $\max _{i, t} \tau_{i}(t)$.

For each node we will use the Lyapunov-Krasovskii functional for time-varying delays $V_{i}\left(t, e_{i, t}, \dot{e}_{i, t}\right)$ corresponding to (4) in the centralized case. Additionally, since we have the delayed errors from incoming neighbors, we exploit Halanay's inequality as is done for the first time in [22]:

Lemma 5. For $i=1, \ldots, N$, assume there exist Lyapunov functions $\underline{k}\left|e_{i}(t)\right|^{2} \leq V_{i}\left(t, e_{i}, \dot{e}_{i}\right) \leq \bar{k}\left\|e_{t, i}\right\|_{W}^{2}$ with $\underline{k}>0$ and $\bar{k}>0, \alpha_{i}>0$ and $\beta_{i, j}>0, j \in \mathcal{N}_{i}$, for which the inequalities

$$
\dot{V}_{i}+2 \alpha_{i} V_{i}-\sum_{j \in \mathcal{N}_{i}} \beta_{i, j} V_{j}\left(t-\tau_{i}(t)\right) \leq 0
$$

hold with $\delta_{0}>\delta_{1}>0$, where $\delta_{0}=\min \alpha_{i}$, and $\delta_{1}=$ $\frac{1}{2} \max _{i} \sum_{j: i \in \mathcal{N}_{j}} \beta_{j, i}$. Then the systems $\left.\sqrt{18}\right|^{i}$ are exponentially 
stable and the convergence rate $\delta$ can be estimated as solutions of (2).

Proof. The above inequality also implies

$$
\dot{V}_{i}+2 \alpha_{i} V_{i}-\sum_{j \in \mathcal{N}_{i}} \beta_{i, j} \sup _{-\bar{\tau} \leq \theta \leq 0} V_{j}(t+\theta) \leq 0 .
$$

We use $W=\sum_{i=1}^{N} V_{i}\left(e_{i}\right)$ as in the delay-free case giving

$$
\begin{array}{r}
\dot{W}=-\sum_{i=1}^{N} 2 \alpha_{i} V_{i}+\sum_{i=1}^{N}\left(\sum_{j: i \in \mathcal{N}_{j}} \beta_{j, i}\right) \sup _{-\bar{\tau} \leq \theta \leq 0} V_{i}(t+\theta) \\
\leq-2 \delta_{0} W+2 \delta_{1} \sup _{-\bar{\tau} \leq \theta \leq 0} W(t+\theta) .
\end{array}
$$

Then from Lemma 1 it follows exponential convergence for the combined error with rate $\delta$ being a solution of (2).

Adding to the inequality (19) the system dynamics via the descriptor method

$$
\begin{aligned}
& 2\left(P_{2, i} e_{i}(t)+P_{3, i} \dot{e}_{i}(t)\right)^{\top} \times\left(\left(A-L_{i} C_{i}\right) e_{i}(t)\right. \\
- & \left.\sum_{j \in \mathcal{N}_{i}} H_{i, j} e_{i}\left(t-\tau_{i}(t)\right)+\operatorname{row}\left\{H_{i, j}\right\}_{j \in \mathcal{N}_{i}} \mathbf{e}_{i}\left(t-\tau_{i}(t)\right)-\dot{e}_{i}\right)=0
\end{aligned}
$$

and similarly to (7), it follows

$$
\dot{V}_{i}+2 \alpha V_{i}-\sum_{j \in \mathcal{N}_{i}} \beta_{i, j} V_{j}\left(t-\tau_{i}(t)\right) \leq \eta_{i}(t)^{\top} \Phi_{i} \eta_{i}(t)
$$

where $\eta_{i}(t)=\operatorname{col}\left\{e_{i}(t), \dot{e}_{i}(t), e_{i}(t-\bar{\tau}), e_{i}\left(t-\tau_{i}(t)\right), \mathbf{e}_{i}(t-\right.$ $\left.\left.\tau_{i}(t)\right)\right\}$,

$$
\begin{array}{ccccc}
\multicolumn{6}{c}{\Phi_{i}=} \\
{\left[\begin{array}{ccccc}
\Xi_{i, 11}+\Psi_{i, 11} & \Xi_{i, 12}+P_{i} & \Psi_{i, 12} & \Xi_{i, 13}+\Psi_{i, 13} & \Xi_{i, 14} \\
* & \Xi_{i, 22}+\bar{\tau}^{2} R_{i} & 0 & \Xi_{i, 23} & \Xi_{i, 24} \\
* & * & \Psi_{i, 22} & \Psi_{i, 23} & 0 \\
* & * & * & \Psi_{i, 33} & 0 \\
* & * & * & * & -\operatorname{diag}\left\{\beta_{i, j} P_{j}\right\} \\
j \in \mathcal{N}_{i}
\end{array}\right]}
\end{array}
$$

with

$$
\begin{aligned}
& \Xi_{i, 11}=\left(A-L_{i} C_{i}\right)^{\top} P_{2, i}+P_{2, i}^{\top}\left(A-L_{i} C_{i}\right) \\
& \Xi_{i, 12}=-P_{2, i}^{\top}+\left(A-L_{i} C_{i}\right)^{\top} P_{3, i} \\
& \Xi_{i, 13}=-P_{2, i}^{\top} \sum_{j \in \mathcal{N}_{i}} H_{i, j} \\
& \Xi_{i, 14}=P_{2, i}^{\top} \operatorname{row}\left\{H_{i, j}\right\}_{j \in \mathcal{N}_{i}} \\
& \Xi_{i, 22}=-P_{3, i}-P_{3, i}^{\top} \\
& \Xi_{i, 23}=-P_{3, i}^{\top} \sum_{j \in \mathcal{N}_{i}} H_{i, j} \\
& \Xi_{i, 24}=P_{3, i}^{\top} \operatorname{row}\left\{H_{i, j}\right\}_{j \in \mathcal{N}_{i}}, \\
& \Psi_{i}=\left[\begin{array}{lll}
\Psi_{i, 11} & \Psi_{i, 12} & \Psi_{i, 13} \\
\Psi_{i, 21} & \Psi_{i, 22} & \Psi_{i, 23} \\
\Psi_{i, 31} & \Psi_{i, 32} & \Psi_{i, 33}
\end{array}\right]= \\
& {\left[\begin{array}{ccc}
2 \alpha_{i} P_{i}+S_{i}-\mathrm{e}^{-2 \alpha_{i} \bar{\tau}} R_{i} & \mathrm{e}^{-2 \alpha_{i} \bar{\tau}} S_{12, i} & \mathrm{e}^{-2 \alpha_{i} \bar{\tau}}\left(R_{i}-S_{12, i}\right) \\
* & -\mathrm{e}^{-2 \alpha_{i} \bar{\tau}}\left(S_{i}+R_{i}\right) & \mathrm{e}^{-2 \alpha_{i} \bar{\tau}}\left(R_{i}-S_{12, i}\right) \\
* & * & \mathrm{e}^{-2 \alpha_{i} \bar{\tau}}\left(-2 R_{i}+S_{12, i}+S_{12, i}^{\top}\right)
\end{array}\right],}
\end{aligned}
$$

from which with Lemma 5 after substituting $P_{3, i}=$ $\epsilon_{i} P_{2, i}, Y_{i}=P_{2, i}^{\top} L_{i}$ and $Z_{i, j}=P_{2, i}^{\top} H_{i, j}$, follows our main result:

Theorem 2. For $i=1, \ldots, N$, given $\bar{\tau}>0,0<\delta_{1}<$ $\delta_{0}$ where $\delta_{0}=\min _{i} \alpha_{i}$, and $\delta_{1}=\frac{1}{2} \max _{i} \sum_{j: i \in \mathcal{N}_{j}} \beta_{j, i}, \alpha_{i}>$ $0, \beta_{i, j}>0$ and tuning variables $\epsilon_{i}$, assume there exist matrices $P_{i}>0, R_{i}>0, S_{i}>0$ and $P_{2, i}, Y_{i}, Z_{i, j}, S_{12, i}$, which satisfy the system of LMIs $\Phi_{i} \leq 0$, with $\Phi_{i}=$

$$
\left[\begin{array}{ccccc}
\Xi_{i, 11}+\Psi_{i, 11} & \Xi_{i, 12}+P_{i} & \Psi_{i, 12} & \Xi_{i, 13}+\Psi_{i, 13} & \Xi_{i, 14} \\
* & \Xi_{i, 22}+\bar{\tau}^{2} R_{i} & 0 & \Xi_{i, 23} & \Xi_{i, 24} \\
* & * & \Psi_{i, 22} & \Psi_{i, 23} & 0 \\
* & * & * & \Psi_{i, 33} & 0 \\
* & * & * & * & -\operatorname{diag}\left\{\beta_{i, j} P_{j}\right\} \\
j \in \mathcal{N}_{i}
\end{array}\right]
$$

$$
\begin{aligned}
& \text { with } \\
& \Xi_{i, 11}=A^{\top} P_{2, i}+P_{2, i}^{\top} A-C_{i}^{\top} Y_{i}^{\top}-Y_{i} C_{i} \\
& \Xi_{i, 12}=-P_{2, i}^{\top}+\epsilon_{i} A^{\top} P_{2, i}-\epsilon_{i} C_{i}^{\top} Y_{i}^{\top} \\
& \Xi_{i, 13}=-\sum_{j \in \mathcal{N}_{i}} Z_{i, j} \\
& \Xi_{i, 14}=\operatorname{row}\left\{Z_{i, j}\right\}_{j \in \mathcal{N}_{i}} \\
& \Xi_{i, 22}=-\epsilon_{i}\left(P_{2, i}+P_{2, i}^{\top}\right) \\
& \Xi_{i, 23}=-\epsilon_{i} \sum_{j \in \mathcal{N}_{i}} Z_{i, j} \\
& \Xi_{i, 24}=\epsilon \operatorname{row}\left\{Z_{i, j}\right\}_{j \in \mathcal{N}_{i}},
\end{aligned}
$$

and $\left[\begin{array}{cc}R_{i} & S_{12, i} \\ * & R_{i}\end{array}\right]>0$. Then the systems 18 with $L_{i}=$ $\left(P_{2, i}^{\top}\right)^{-1} Y_{i}$ and $H_{i, j}=\left(P_{2, i}^{\top}\right)^{-1} Z_{i, j}$ converge exponentially for any delay $0 \leq \tau_{i}(t) \leq \bar{\tau}$ with convergence rate $\delta$ obtained as a solution of (2).

Remark 3. Our results can be extended to the case of disturbances in the dynamics and measurements, leading to input-to-state stability of the estimation error. This may be a topic for future research.

\section{EXAMPLE}

To verify the presented methods the design of a centralized observer and a distributed observer is carried out on a numerical example and tested in a simulation with a sawtooth shaped delay. Moreover, the two designs are compared in three different ways. The feasibility of the LMIs used for the design gives a sufficient bound, which is however more conservative than the bounds obtained by the LMIs for analysis (i.e. without variable substitution). Lastly, the bounds obtained with the simulations are compared. This is done for the same convergence rate to ensure that the designs are comparable.

The numerical example is adapted from [1]. The system dynamics and one output have been changed so that the system is unstable $\left(\lambda_{\max }(A)=1\right)$ and no single observer node is detectable (i.e. the pairs $\left(A, C_{i}\right), i=1, \ldots, N$ are 


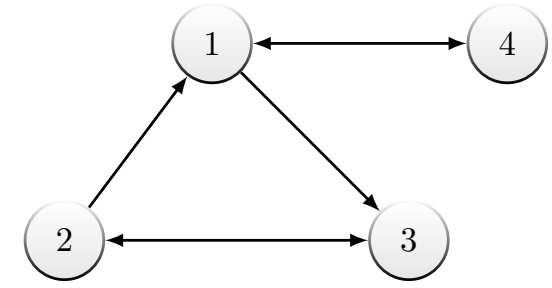

Fig. 2: Communication graph of the distributed observer.

not detectable). The communication graph for the distributed observer is kept the same and is shown in Fig. 2.

$$
\begin{aligned}
A= & {\left[\begin{array}{cccccc}
-1 & 0 & 0 & 0 & 0 & 0 \\
-1 & 1 & 1 & 0 & 0 & 0 \\
1 & -2 & -1 & -1 & 1 & 1 \\
0 & 0 & 0 & 1 & 0 & 0 \\
-8 & 1 & 1 & -1 & -2 & 0 \\
4 & -0.5 & 0.5 & 0 & 0 & -4
\end{array}\right] } \\
C_{1} & =\left[\begin{array}{cccccc}
1 & 0 & 0 & 2 & 0 & 0 \\
2 & 0 & 0 & 1 & 0 & 0
\end{array}\right] \\
C_{2} & =\left[\begin{array}{llllll}
2 & 0 & 1 & 0 & 0 & 0
\end{array}\right] \\
C_{3} & =\left[\begin{array}{llllll}
0 & 0 & 0 & 2 & 0 & 0
\end{array}\right] \\
C_{4} & =\left[\begin{array}{llllll}
1 & 0 & 2 & 0 & 0 & 0 \\
2 & 0 & 4 & 0 & 0 & 0
\end{array}\right] .
\end{aligned}
$$

Centralized observer: The observer gain $L$ is designed according to Proposition 11 with YALMIP [23] as solver for the LMI. The convergence rate is first fixed to $\alpha=1$, then $\bar{\tau}$ is maximized while consecutively adapting $\epsilon$. The maximal possible $\bar{\tau}$ is found to be 0.204 with $\epsilon=0.5$. Then (8) with (6) is found feasible for $\bar{\tau}=0.2916$ for $\alpha=1$ and for $\bar{\tau}=0.304$ for $\alpha=0$. The design is verified in a simulation where the measurement input is sampled with a rate of $\bar{\tau}^{-1}$, showing that the desired rate of convergence is attained (Fig. 3. Next the sampling rate is gradually decreased, showing that from $\bar{\tau}=0.430$ the convergence rate will be less than 1 (Fig. 4). From $\bar{\tau}=0.494$ the error will start to diverge; the observer becomes unstable.

Distributed observer: To simplify the design we choose $\alpha_{i}, \beta_{i, j}$ and $\epsilon_{i}$ the same for all nodes. Then for the desired convergence rate $\delta=1$ we obtain from (2)

$$
\delta_{1}=\frac{\delta_{0}-\delta}{\mathrm{e}^{2 \delta \bar{\tau}}}
$$

which fixes $\beta_{i, j}$. As before, $\bar{\tau}, \alpha_{i}$ and $\epsilon_{i}$ are consecutively adapted and Theorem 2 is applied. The LMI is found feasible for $\bar{\tau}=0.226, \alpha_{i}=1.14$ and $\epsilon_{i}=0.419$. With the designed $L_{i}, H_{i, j}$ the centralized analysis LMI (8), (6) can be used with $\alpha=1$ and is found feasible for $\bar{\tau}=0.243(\bar{\tau}=0.354$ for $\alpha=0)$.

In the simulation with the same sawtooth shaped timevarying delay for all communications (i.e. modeling sampleddata communication with equal rate), the convergence rate is breached for $\bar{\tau}=0.525$ and it diverges for $\bar{\tau}=0.625$. Table I summarizes the comparison of the centralized and the distributed observer, Fig. 5 and Fig. 6 show the coordinates of the error in linear scaling.

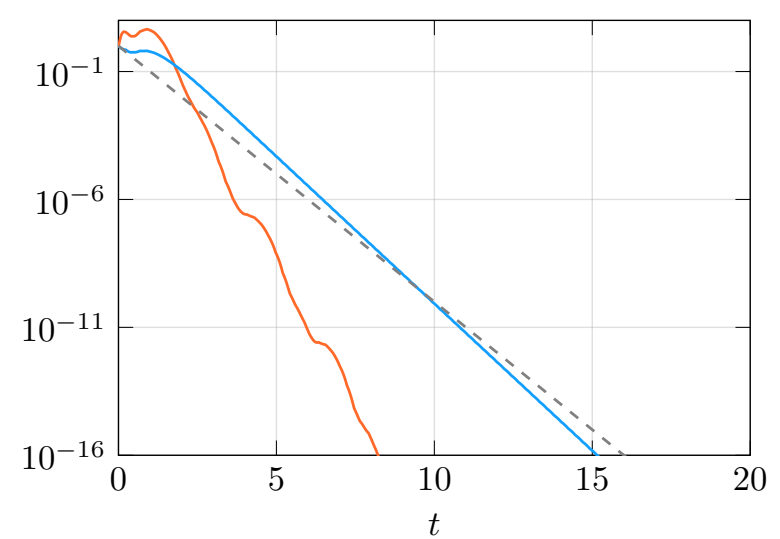

Fig. 3: Error norm in logarithmic scaling (red: Distributed, blue: Centralized) for $\bar{\tau}=0.226$.

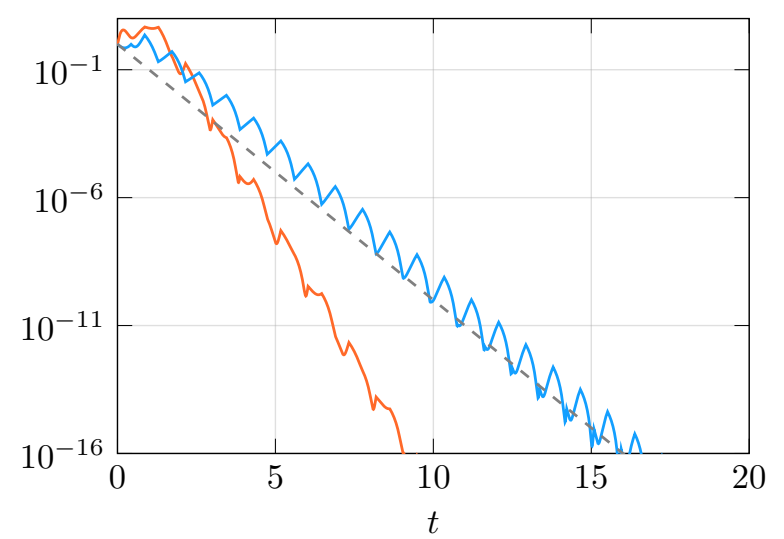

Fig. 4: Error norm in logarithmic scaling (red: Distributed, blue: Centralized) for $\bar{\tau}=0.430$.

\section{Conclusion}

In this paper, two new designs of distributed observers were proposed for delay-free and a time-varying delay cases. The obtained conditions for observer gain calculation and stability analysis were formulated in terms of LMIs. The convergence rate of the estimation error was evaluated (it is a parameter of the LMIs).

To highlight the advantages of the distributed setting of estimation over the centralized one, a design was performed under reasonable assumptions and a comparison in numerical experiments for both settings were carried out. The comparison showed that for the selected example, the maximal allowable delay is higher for the distributed observer (the results based on the feasibility of the LMIs and the simulations are consistent) since the centralized observer loses performance faster than the distributed one.

This also points to the conception, that in the distributed setting, it is more beneficial for the observer nodes to exchange state estimates than directly the measurements. A plain explanation of this is that there are more degrees of freedom in the consensus gains than in the output injection gains, due to the fact that the outputs are lower-dimensional maps of the state. However the transmission of full state-estimates between the nodes might not be feasible for a high-dimensional plant. Note 
that following the proof of Lemma 3, the nodes could send the projection to the unobservable subspace of the receiving node instead of the full state-estimate and on the example, it can be verified that this does not affect the achievable performance (since the observable subspace can be reconstructed solely from the delay-free measurement output). Therefore, a future research direction is to see how the benefits of using consensus coupling are prevailed if some lower-dimensional artificial outputs are exchanged instead of the full state estimates (ultimately, the choice of the artificial output will lead to a trade-off with respect to the communication costs).

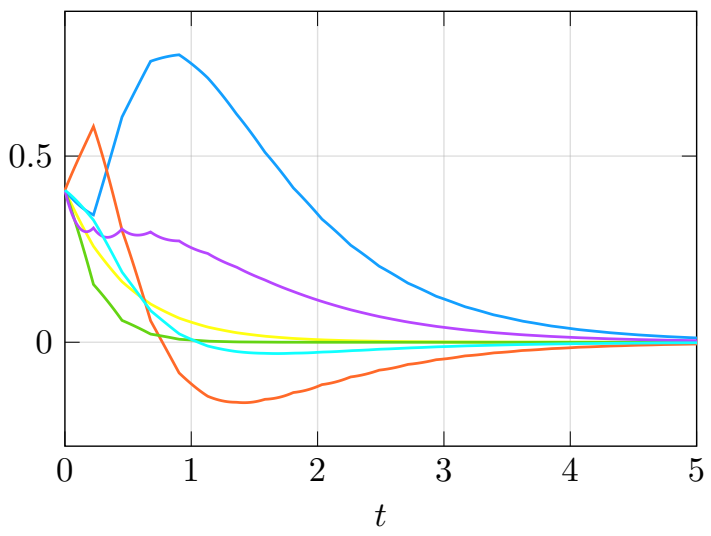

Fig. 5: Error of each node of the centralized observer for $\bar{\tau}=$ 0.226 .

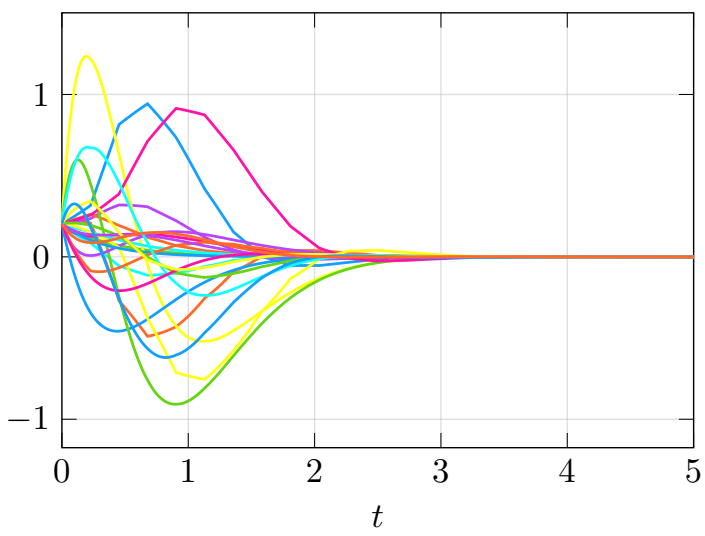

Fig. 6: Error of each node of the distributed observer for $\bar{\tau}=$ 0.226 .

\begin{tabular}{lcc}
\hline & centralized & distributed \\
\hline for convergence rate 1 & \\
design & 0.204 & 0.226 \\
analysis & 0.204 & 0.243 \\
simulation & 0.430 & 0.525 \\
without prescribed rate & (i.e. & $\alpha=0)$ \\
analysis & 0.304 & 0.354 \\
simulation & 0.494 & 0.625 \\
\hline
\end{tabular}

TABLE I: Summary of maximal obtained $\bar{\tau}$ and simulation result with sawtooth shaped delay for the centralized and the distributed observer.

\section{REFERENCES}

[1] W. Han, H. L. Trentelman, Z. Wang, and Y. Shen, "A Simple Approach to Distributed Observer Design for Linear Systems," IEEE Transactions on Automatic Control, vol. 64, no. 1, pp. 329-336, Jan. 2019.

[2] Á. R. del Nozal, P. Millán, L. Orihuela, A. Seuret, and L. Zaccarian, "Distributed estimation based on multi-hop subspace decomposition," Automatica, vol. 99, pp. 213-220, Jan. 1, 2019.

[3] A. Mitra and S. Sundaram, "Distributed observers for LTI systems," IEEE Transactions on Automatic Control, vol. 63, no. 11, pp. 36893704, Nov. 2018.

[4] L. Wang and A. S. Morse, "A distributed observer for a time-invariant linear system," IEEE Transactions on Automatic Control, vol. 63, no. 7, pp. 2123-2130, Jul. 2018.

[5] V. Ugrinovskii, "Distributed robust filtering with $\mathrm{H} \infty$ consensus of estimates," Automatica, vol. 47, no. 1, pp. 1-13, Jan. 1, 2011.

[6] F. F. C. Rego, A. M. Pascoal, A. P. Aguiar, and C. N. Jones, "Distributed state estimation for discrete-time linear time invariant systems: A survey," Annual Reviews in Control, vol. 48, pp. 36-56, 2019.

[7] P. Millán, L. Orihuela, C. Vivas, and F. R. Rubio, "Distributed consensus-based estimation considering network induced delays and dropouts," Automatica, vol. 48, no. 10, pp. 2726-2729, Oct. 1, 2012.

[8] P. Millán, L. Orihuela, I. Jurado, C. Vivas, and F. R. Rubio, "Distributed estimation in networked systems under periodic and event-based communication policies," International Journal of Systems Science, vol. 46, no. 1, pp. 139-151, Jan. 2, 2015.

[9] H. Basu and S. Y. Yoon, "Distributed state estimation by a network of observers under communication and measurement delays," Systems \& Control Letters, vol. 133, p. 104554 , Nov. 1, 2019.

[10] K. Liu, J. Lü, and Z. Lin, "Design of Distributed Observers in the Presence of Arbitrarily Large Communication Delays," IEEE Transactions on Neural Networks and Learning Systems, vol. 29, no. 9, pp. 4447-4461, Sep. 2018.

[11] E. Fridman, A. Seuret, and J.-P. Richard, "Robust sampled-data stabilization of linear systems: An input delay approach," Automatica, vol. 40, no. 8, pp. 1441-1446, Aug. 1, 2004.

[12] V. Ugrinovskii and E. Fridman, "A round-robin type protocol for distributed estimation with $\mathrm{h} \infty$ consensus," Systems \& Control Letters, vol. 69, pp. 103-110, Jul. 1, 2014.

[13] F. Dörfler, F. Pasqualetti, and F. Bullo, "Continuous-time distributed observers with discrete communication," IEEE Journal of Selected Topics in Signal Processing, vol. 7, no. 2, pp. 296-304, Apr. 2013.

[14] H. Silm, R. Ushirobira, D. Efimov, W. Michiels, J.-P. Richard, and E. Fridman, "Comparison of the time-delay margin of a distributed and centralized observer," in 2018 European Control Conference (ECC), Limassol, Cyprus, Jun. 2018, pp. 1963-1968.

[15] H. K. Khalil, Nonlinear Systems. Prentice Hall, 2002.

[16] E. Fridman, Introduction to Time-Delay Systems: Analysis and Control. Birkhäuser Basel, 2014.

[17] A. Halanay, Differential Equations: Stability, Oscillations, Time Lags. Academic Press, Jan. 1, 1966.

[18] P. Park, J. W. Ko, and C. Jeong, "Reciprocally convex approach to stability of systems with time-varying delays," Automatica, vol. 47, no. 1, pp. 235-238, Jan. 1, 2011.

[19] R. Olfati-Saber, "Distributed Kalman filtering for sensor networks," in 2007 46th IEEE Conference on Decision and Control, Dec. 2007, pp. 5492-5498.

[20] A. Sootla, Y. Zheng, and A. Papachristodoulou, "On the existence of block-diagonal solutions to Lyapunov and $\mathrm{H} \infty$ Riccati inequalities," IEEE Transactions on Automatic Control, vol. 65, no. 7, pp. 31703175, 2020.

[21] L. Wang, J. Liu, and A. S. Morse, "A distributed observer for a continuous-time linear system," in 2019 American Control Conference (ACC), Jul. 2019, pp. 86-91.

[22] E. Fridman and A. Blighovsky, "Robust sampled-data control of a class of semilinear parabolic systems," Automatica, vol. 48, no. 5, pp. 826836, May 1, 2012.

[23] J. Lofberg, "YALMIP : A toolbox for modeling and optimization in MATLAB," in 2004 IEEE International Conference on Robotics and Automation, vol. Halan, Sep. 2004, pp. 284-289. 